\title{
Research on the Multimedia Information Retrieval Algorithm based on Information Restructuring and Image Reconstruction
}

\author{
Nian Li \\ Wuhan Donghu University ,No.301, Wenhuaroad,Jiangxia, \\ Wuhan,Hubei, China,430212
}

\begin{abstract}
In this paper, we conduct research on the multimedia information retrieval algorithm based on the information restructuring and image reconstruction. With the massive growth of information resources, people through various retrieval tools for too much information, led directly to information overload. In vector space model and probability retrieval model based on information retrieval tools rarely consider the user's personalized information needs and features, has resulted in a large amount of information retrieval result and correlation information the user's information demand is not big. In order to improve the existing retrieval system, in recent years, scholars to study looked that context information retrieval context factors need to be considered, such as the retrieval time, place and the interactive history, mission, environment and other factors stated or implied in the retrieval process. At present, the context research has become the information behavior, information search process and the research hotspot in the field of information retrieval interaction.
\end{abstract}

Keywords- Multimedia Information, Retrieval Algorithm, Image Reconstruction, Data Analysis.

\section{Introduction}

It is the basic principle of computer information retrieval by a computer to question word compared with key words, find out the information in the consistent with the question word search word, and according to the key words will draw on the information from the database, provides to the user as it is through the computer retrieval machine-readable information directory [1-3].

With the popularity of the Internet and the development of e-commerce, enterprise and individual is the amount of information available, need to handle in explosive growth, some new problems have sprung up information retrieval, this makes it necessary to use the information visualization analysis technology, intuitively showing knowledge source, the main body of information retrieval research, research hot spot and front that make readers to the retrieval research interest to have a more clear understanding. According to the literature review, the primary information retrieval methods could be summarized as follows. (1) Intelligent information retrieval. Intelligent information retrieval is the general combination of the artificial intelligence and information retrieval technology is applied to the achievements in the field of information access. Intelligent information retrieval based on natural language retrieval, machine based on natural language retrieval requirements provided by the user is analyzed, and then form a retrieval strategy. It is able to replace or assist users, such as word choice, choose library, retrieval type structure, even in database to find functions such as automatic reasoning. (2) Visual information retrieval. Compared with other information retrieval technology, advantages of visual information retrieval technologies is the understanding of literature or retrieval type internal semantic relations and help users determine a search related literature. (3) Cross-media information retrieval. Compared with the retrieval method based on the content, the retrieval mechanism not only can obtain more abundant retrieval results, and as far as possible to use various knowledge to further improves the relevance of the retrieved results is a very active 
relative to the conservative retrieval mechanism. (4) Personalized information retrieval. Users can change the format of the basic retrieval results show that to some extent, the user can also choose their own sources of information, ask all kinds of users are satisfied with their own sources, for certain types of information, and users can also further limits, the result of the hit requirements will only provide authoritative and reliable results, and thus improve the precision, resolution and can meet the needs of different users retrieval function, can even understand different users different working style and show the information to the user in the application of the basic custom and haute couture [4].

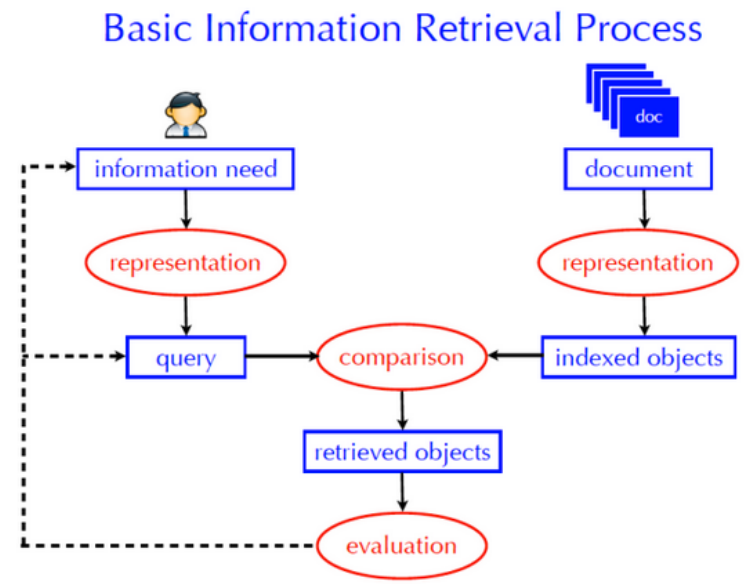

Figure 1. The General Procedures of the Information Retrieval Process

In this paper, we conduct research on the multimedia information retrieval algorithm based on the information restructuring and image reconstruction. Along with the development of the information age, multimedia information at a speed of index growth, at the same time appear more and more abundant information types, such as static images, graphics, audio, and video and so on. In the face of the huge amounts of multimedia information, must have the corresponding retrieval techniques to identify and capture the required information, and bring them out, become the "useful" information for specific users. At present, there are two main types of multimedia retrieval technology: text-based retrieval and content-based retrieval technology. In the later sections, we will discuss in detail.

\section{Our Proposed Methodology}

The Image Reconstruction. Based on Shannon sampling theorem, the traditional signal processing method is mainly from aspects of improving the sampling frequency or limitation of signal bandwidth research. But the process of the first high-speed sampling again compressed store waste a great deal of sampling resources, there is larger redundancy. Therefore, signal description and the basic processing research to establish a new framework, under the framework to achieve far below traditional of signal sampling, sampling rate in order to reduce amount of data collected, facilitate remote transmission, and can accurately reconstruct the signal which has the vital significance [5-6].

Signal reconstruction is one of the core issues of compressed sensing theory this article focuses on signal reconstruction method based on greedy algorithm as greedy algorithm is an iterative algorithm, it through the repeated iteration in the target solution space search, until we find a satisfactory solution or the maximum number of the iterations. Greedy algorithm reconstruction is very fast, but some characteristics of reconstruction effect depends on the signal itself, with the position of the nonzero elements distribution in sparse signal under the condition of not clear, can't choose one of the best algorithm for signal reconstruction. In addition, the measured value of the greedy algorithm requires a minimum, once the lower than the measured value, the reconstruction effect of the algorithm will be greatly reduced. The primary framework could be summarized as the follows.

$$
\min \|s\|_{0} \quad \text { s.t. } \quad\|\Phi s=y\|_{2} \leq \varepsilon
$$

To solve the above problems, this paper presents a new fusion framework, under the framework of existing two kinds of greedy 
algorithm fusion, through these two kinds of algorithms of support set of occurring simultaneously set processing, choose the most suitable atom to reconstruct the original signal, realize the support set of secondary screening, finally realizes the accurate reconstruction of the signal, the integration framework, the choice of algorithm can also be replaced by the other greedy algorithm. With the data obtained through coordinate transformation is original depth information, this information is not color, texture rendering, it is difficult to identify, to the depth of field image processing more difficult. In the computer graphics, a polygon surface model because of its simple, flexible features, is the most commonly used a method of 3D object model representation and triangle surface model is the most basic polygon surface model representation. Each frame is shown there are a lot of small triangle patchworks and plane normal vector can reflect different flat position, according to the characteristic according to different values of each plane normal vector on each point coloring, so it can effectively show the characteristics of different plane [7].

The Multimedia Information Retrieval. Content-based image retrieval is mainly using the underlying characteristics of images, such as color, texture, shape, and spatial relationships and other features for image retrieval, trying to understand the image content searching in accordance with user requirements on the basis of the image. Color is the most significant characteristics of the underlying image. The classic retrieval algorithm is the global color histogram method, this method is quick and easy, and rotation, translation and scaling of the images are not sensitive to changes, but it can't reflect the color spatial location information. For example, the two completely different images may have the same color histogram and this will cause the error of retrieval.

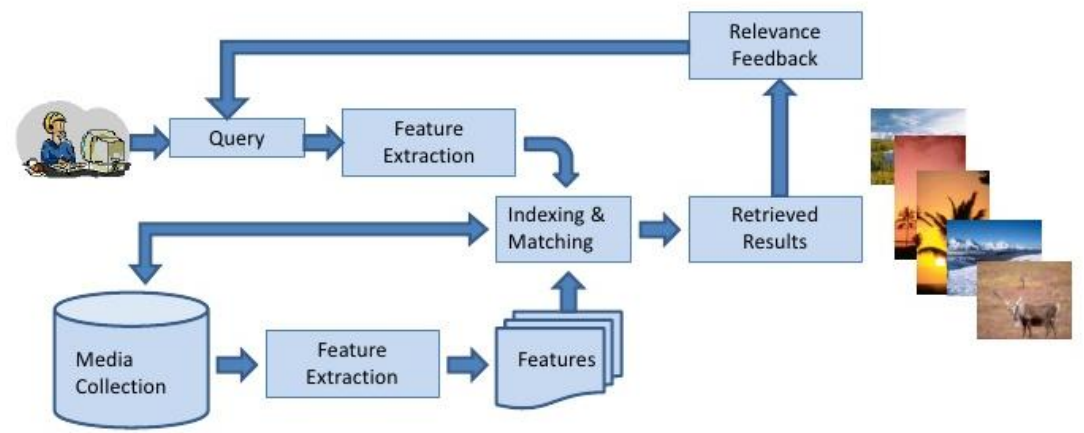

Figure 2. The Demonstration of the Classic Multimedia Information Retrieval System

Accumulative histogram can be avoided in the global color histogram unable to get through all of color characteristic value and appear some of the zero value, improve the retrieval accuracy. In image retrieval, have proved that accumulative histogram is global histogram retrieval effect is good. Based on block of color histogram is according to some kind of cross molecular block strategy divided the whole image into several blocks, and then calculate the color information of each block although these feature extraction includes spatial information of the image, but at the same time, inevitably, destroyed the original algorithm of rotation, translation and scaling invariance, so for image retrieval and no good retrieval effect. For this drawback, we propose the revised distance standard as follows.

$$
D_{i}=\sum_{i=1}^{n} \sum_{j=1}^{m}\left|I_{p}(i j)-I_{Q}(i j)\right|^{1 / 2}
$$

In this paper, the similarity measure of color features using the Euclidean distance method. The statistics of similar distance between each block, and endow the different weights for different block, will be the sum of weighted distance after as the actual distance between two 
images as the smaller the actual distance, the more similar two pictures [8].

The Information Retrieval Framework. Computer retrieval as an advanced means of utilization of global information resources management and decision service function has been used more and more people know, compared with the manual retrieval, it has the following advantages. (1) Quick lookup and timely feedback, resource sharing. As the central mainframe uses time-sharing technology in the online system, the system response to the user instructions are usually only a few seconds, retrieval feedback quickly, according to the feedback of the system, adjust the retrieval strategy at any time, and really made it the world's information resource sharing network retrieval, the user terminal can be installed in the office or at home through the Internet contact with all kinds of literature database center, to obtain the required documents. (2) Easy to use, fully functional, with flexible piece together. Computer information retrieval using the Boolean logic operation, flexible between various retrieval words can piece together that can also be to retrieve the location of the relationship between full-text search and phrase. (3) Retrieve comprehensive, the real-time theme is extensive, is strong. Online retrieval often provides tens of to hundreds of database retrieval and the design theme is very extensive, almost covers all areas of human social life every time new information on the Internet that may at any time to find and get the latest information.

Based on the mentioned research, we could categorize the corresponding issues as follows. (1) The complexity of the task. With the increasingly complex of retrieval task and deepening, some scientific research content has been gradually showing a trend of interdisciplinary, across domains and across the organization, and breakthrough the limitation of the time, space and geography. In this context, the user power alone to complete complex retrieval task is increasingly difficult, everyone needs to set each specialty. (2) The complexity of information needs. The user's information demand expression is associated with the background of the user as the user's knowledge structure determines the demand for information understanding and general expression. User demand information only match with its knowledge structure that can be understood and absorbed. (3) Cognitive complexity. Because the user level of education, the knowledge structure are different, their professional knowledge, thinking mode, preferences, expectations and evaluation, etc. also each are not identical, different users in the collaborative retrieval has great difference in cognition. Individual users can work together to solve the problem of insufficient knowledge structure and ability to interact in the process of the personal characteristic, the cognitive style and emotional status to the team's overall cognitive that make group cognitive complexity [9].

The Information recombination. The information restructuring is an important concept of the information organization science, it is a comprehensive utilization of the information description and records, information revealed and the sorting, information indexing, information storage and analysis and research methods such as processing and low level of information for identification, screening, analysis, extraction, enrichment and the whole sequence to form a higher level the process of the information products. Information reorganization, in other words, is not only the order information from unordered to certain goals, from mixed to sophisticated information of ordering and the good process, is also a pursuit, the whole sequence information environment and in accordance with the specific goal to build all kinds of new type of the information products and information access system, accelerate the process of effective development and utilization of information that can be listed as:

- Deconstruction of basic information. Refers to the deep analysis of the specific 
information knowledge content, on the basis of the decomposition in the information in time and space. Information of deconstruction results will get some letter.

- Potential of information. It is the information of "potential energy" as it is measure of the value of information. Information on the potential of decision of the size of the information flow of the power. Information potential is higher, to the letter, the greater the value, the greater the power of the flow of information.

- Information value. Information itself has value, and can realize the value added in the flow. Information value-added performance for the increase of the potential, this is a process of the office, the increment is derived from information workers labor, is the crystallization of value.

- Information. According to the information contained in, based on the knowledge content into information is divided into unit, we call this information. The information can contain several units and these information units can have different combinations [10].

The restructuring of the implicit information is essentially the hidden knowledge exists in a variety of media unit, information unit excavated and clustering set into the knowledge base, database to compare, analysis and research, into a man's wisdom, output of new process of creative knowledge. It involves the content and form diversification, stability and variability, and some control disciplines and specialties, the trend of the research direction of power. With the thinking of the human brain, and highly personalized, at the same time, according to specific circumstances, specific areas, specialized knowledge, technology, comprehensive analysis and research, forming related new high value-added information and knowledge. Development, restructuring the implicit knowledge unit, information unit is often the key to innovation with invention and discovery.

\section{Conclusion}

In this paper, we conduct research on the multimedia information retrieval algorithm based on the information restructuring and image reconstruction. Video to modern content-based image retrieval technology is a hotspot of research on the general video image processing technology in recent years. Content-based image retrieval method mainly divides into the global method and local characteristics. Global features method, and difficult to extract and express effectively, it can only be used in some special occasions; Local characteristics method processing of information is less, and characteristics of the obvious, so widely used. Traditional content-based image retrieval and the traditional semantic image retrieval method can well depict image contains rich semantic information, also can't good use text retrieval has the ability of complete semantic expression. Its core problem is how to effectively in a text semantic space and establish an effective mapping relationship between image feature spaces. In the future, we will conduct more research for optimization.

\section{References}

[1] Benavent, Xaro, et al. "Multimedia information retrieval based on late semantic fusion approaches: Experiments on a wikipedia image collection." Multimedia, IEEE Transactions on 15.8 (2013): 2009-2021.

[2] Eskevich, Maria, et al. "Multimedia information seeking through search and hyperlinking." Proceedings of the 3rd ACM conference on International conference on multimedia retrieval. ACM, 2013. 
[3] Carpineto, Claudio, and Giovanni Romano. "A survey of automatic query expansion in information retrieval." ACM Computing Surveys (CSUR) 44.1 (2012): 1 .

[4] Wang, Yanqiu, XiaoFei Yan, and Wei Zhang. "An Algorithm of Feather and Down Target Detection and Tracking Method Based on Sparse Representation." Measuring Technology and Mechatronics Automation (ICMTMA), 2015 Seventh International Conference on. IEEE, 2015.

[5] Liao, Zicheng, et al. "A subdivision-based representation for vector image editing." Visualization and Computer Graphics, IEEE Transactions on 18.11 (2012): 1858-1867.

[6] Wang, Haoxiang, and Jingbin Wang. "An effective image representation method using kernel classification." Tools with Artificial Intelligence (ICTAI), 2014 IEEE 26th International Conference on. IEEE, 2014.
[7] Meng, Tao, et al. "Enhancing multimedia semantic concept mining and retrieval by incorporating negative correlations." Semantic Computing (ICSC), 2014 IEEE International Conference on. IEEE, 2014.

[8] Wang, Xiang-Yang, Hong-Ying Yang, and Dong-Ming Li. "A new content-based image retrieval technique using color and texture information." Computers \& Electrical Engineering 39.3 (2013): 746-761.

[9] Roelleke, Thomas. "Information retrieval models: Foundations and relationships." Synthesis Lectures on Information Concepts, Retrieval, and Services 5.3 (2013): 1-163.

[10] Gallego, Mario Arias, et al. "Compressing semantic metadata for efficient multimedia retrieval." Advances in artificial intelligence. Springer Berlin Heidelberg, 2013. 12-21. 